\title{
Lessons Learned from Two Missed Prenatal Cases of Hemoglobin Bart's Hydrops Fetalis Until Second Trimester Despite a Nationwide Screening Program
}

\author{
Wu WJ',2\#, Ma GC ${ }^{1,3 \#}$, Wu PC ${ }^{4}$, Huang KS ${ }^{5}$, Liu YL ${ }^{5}$, Chang SP ${ }^{1}$, Ginsberg NA ${ }^{6}$ and Chen M $^{1,2,7,8 *}$ \\ ${ }^{1}$ Department of Genomic Medicine, and Department of Genomic Technology and Science, Changhua Christian Hospital, Changhua, Taiwan \\ ${ }^{2}$ Department of Obstetrics and Gynecology, Changhua Christian Hospital, Changhua, Taiwan \\ ${ }^{3}$ Institute of Biochemistry, Microbiology and Immunology, Chung-Shan Medical University, Taichung, Taiwan \\ ${ }^{4}$ Taiji Fetal Medicine Center, Taipei, Taiwan \\ ${ }^{5}$ Department of Obstetrics and Gynecology, Tri-Service General Hospital, National Defense Medical Center, Taipei, Taiwan \\ ${ }^{6}$ Department of Obstetrics and Gynecology, Feinberg School of Medicine, Northwestern University Medical Center, Chicago, IL, USA \\ ${ }^{7}$ Department of Obstetrics and Gynecology, College of Medicine, and Hospital, National Taiwan University, Taipei, Taiwan \\ ${ }^{8}$ Department of Life Science, Tunghai University, Taichung, Taiwan \\ \#These authors contributed equally to this study
}

\begin{abstract}
Hemoglobin $(\mathrm{Hb})$ Bart's hydrops fetalis is the most severe form of alpha-thalassemia and has high mortality. It is caused by deletion of all four $\alpha$-globin genes leading to a severe deficiency in a-globin and to the production of $\mathrm{y}$-globin tetramers, resulting in ineffective tissue oxygen delivery. Current preconceptional or prenatal screening policies for this disorder are comprehensive, but not all pregnancies with $\mathrm{Hb}$ Bart's disease can be detected early before hydrops become apparent. We report here two cases with $\mathrm{Hb}$ Bart's disease that escaped being detected until second trimester after hydrops fetalis occurred. These cases remind the clinicians that the importance of this well-characterized condition (alpha-thalassemia) should be reasserted despite a nationwide screening program is available. We also review the current status of prenatal screening and diagnosis of alpha-thalassemia.
\end{abstract}

Keywords: Alpha-thalassemia; Hb Bart's; Hydrops fetalis; Fetal ascites; Cardiomegaly

Abbreviations: Hb: Hemoglobin; SEA: Southeastern Asia; HbCS: $\mathrm{Hb}$ Constant Spring; HbQS: Hb Quong Sze; GA: Gestational Age; MCV: Mean Corpuscular Volume; CBC: Complete Blood Count; MCH: Mean Corpuscular Hemoglobin; MCA-PSV: Peak Systolic Velocity of Middle Cerebral Artery; CTR: Cardiothoracic Ratio; MoM: Multiples of Median; IHUV Vmax: Intrahepatic Umbilical Venous Maximum Flow Velocity; ARMS: Amplification Refractory Mutation System; PCR: Polymerase Chain Reaction

\section{Introduction}

Alpha-thalassemia is one of the most common hereditary hemoglobinopathies, which interferes with the production of $\alpha$-globin chains of hemoglobin $(\mathrm{Hb})$ and causes perinatal complications. Approximately $5 \%$ of the world's population carries mutant or variant alleles over the a-globin gene, with the high frequencies throughout most of Southeastern Asia (SEA), the Mediterranean area, the Indian subcontinent, the Middle East and Africa. In SEA region, the frequency of $\alpha^{0}$-thalassemia variants ( $\left.\alpha \alpha /--\right)$ is about $5-15 \%$ [1]. Among Taiwanese, the carrier rate of alpha-thalassemia is approximately 5-7\% [2]. The disorder is associated with more than 100 known molecular defects in the gene cluster located on the short arm of chromosome 16 (16p13.3) and these defects can result in various clinical phenotypes [3]. Hb Bart's hydrops fetalis is the most severe form of alpha-thalassemia, which is caused by deletion of all four $\alpha$-globin genes (- -/- -), and therefore no $\alpha$-globin is produced. As a result, $\gamma$-globin tetramers (Hb Bart's) become the dominant form of $\mathrm{Hb}$ and are responsible for carrying oxygen from mother to fetus. However, Hb Bart's has a characteristic of extremely high oxygen affinity, which prevents oxygen release from the red blood cells to the tissue. This results in the occurrence of hydrops fetalis, stillbirth, or neonatal demise. Hence, it is critical to diagnose $\mathrm{Hb}$ Bart's hydrops fetalis in early pregnancy as well as to identify high-risk groups for hereditary hemoglobinopathies. However, despite the current nationwide preconceptional or prenatal screening policies, not all pregnancies with $\mathrm{Hb}$ Bart's disease can be detected in the first or early second trimester before hydrops become apparent. Here, we report two prenatal cases of $\mathrm{Hb}$ Bart's disease, which escaped being detected by current screening program until second trimester after developing hydrops fetalis. Besides, we discuss the limitations of current "comprehensive" nationwide screening program used to screen alpha-thalassemia, as well as to review the current status and the future prospects.

\section{Case Report}

\section{Patient 1}

A 33-year-old primigravida was referred to our hospital at $20^{+6}$ weeks of gestational age (GA) after her obstetrician noted fetal ascites in routine antenatal care. The parents of the fetus were both Han Chinese. In the first visit to our hospital, the mother claimed that she had normal antenatal test results except iron deficiency anemia by the result of complete cell count (CBC), ferritin, serum iron and total iron binding capacity. But her obstetricians didn't request her husband to be checked. The initial ultrasound performed at our hospital showed a live singleton fetus whose estimated fetal weight below the 10th percentile. Anatomical examination demonstrated massive fetal ascites, echogenic bowels and cardiomegaly (cardiothoracic ratio: 0.63 ) (Figure 1). No other structural abnormalities were noted. The amniotic fluid volume

*Corresponding authors: Ming Chen, Department of Genomic Medicine Changhua Christian Hospital, Changhua, Taiwan, Fax: 88647249847; E-mail: mingchenmd@gmail.com; mchen_cch@yahoo.com

Received September 24, 2015; Accepted October 23, 2015; Published October 26 2015

Citation: Wu WJ, Ma GC, Wu PC, Huang KS, Liu YL, et al. (2015) Lessons Learned from Two Missed Prenatal Cases of Hemoglobin Bart's Hydrops Fetalis Until Second Trimester Despite a Nationwide Screening Program. Genetics S7: 003. doi:10.4172/2161-1041.S7-003

Copyright: @ 2015 Wu WJ, et al. This is an open-access article distributed under the terms of the Creative Commons Attribution License, which permits unrestricted use, distribution, and reproduction in any medium, provided the original author and source are credited. 
was within normal limits and peak systolic velocity of middle cerebral artery (MCA-PSV) was below 1.5 multiples of median (MoM). We performed amniocentesis and rechecked parental blood samples to exclude the possibility of chromosome abnormality and intrauterine infections. All of the tests were normal, but both of the parents showed microcytic anemia (Mother: $\mathrm{Hb}: 10.6 \mathrm{~g} / \mathrm{dL}$, mean corpuscular volume (MCV): $69.5 \mathrm{fL}$; Father Hb: $11.6 \mathrm{~g} / \mathrm{dL}, \mathrm{MCV}: 69.0 \mathrm{fL})$. We highly suspected that the etiology of fetal ascites was Hb Bart's disease. The DNA from the couple (from peripheral blood) and the fetus (from amniotic fluid) were thus sent for genetic testing. The results revealed that both parents are carriers of alpha-thalassemia $\left(\alpha \alpha /{ }_{-}{ }^{\text {SEA }}\right)$ and the fetus is a case of $\mathrm{Hb}$ Bart's hydrops fetalis $(\zeta \zeta--/ \zeta \zeta--)$. After non-directive genetic counseling and consultation of neonatal and pediatric hematological specialists, the parents elected to terminate the pregnancy.

\section{Patient 2}

A 28-year-old primigravida admitted into our hospital at GA $24^{+1}$ weeks because of acute upper respiratory tract infection. The admission surveillance incidentally showed cardiomegaly (cardiothoracic ratio: 0.68). Reviewing her personal history, she was a victim of alphathalassemia trait. However, her husband was considered as a noncarrier of alpha-thalassemia only by means of electrophoresis. No genetic testing was performed for DNA studies of thalassemia. The parents of the fetus were both Han Chinese. Detailed ultrasound showed no other anatomical abnormality except echogenic bowels. The MCAPSV was below 1.5 MoM. Clinical surveillance including intrauterine infection screening, $\mathrm{Hb}$ genotyping and array comparative genomic hybridization was performed for the couple and fetus. As a result, both of the parents were demonstrated to be carriers of alpha-thalassemia $\left(\alpha \alpha /\right.$ - $\left.^{\text {SEA }}\right)$, and the fetus was proven to be a case of hydrops fetalis due to $\mathrm{Hb}$ Bart's disease by DNA studies. Other investigation revealed normal result except an irrelevant balanced translocation was found: $46, X X, t(1 ; 4)(\mathrm{p} 32.1 ; \mathrm{q} 31.3)$. After explanation of the possible perinatal outcome to the parents, they requested to terminate the pregnancy.

\section{Discussion}

In this report, we present two prenatal cases of Hb Bart's disease that escaped early detection until second trimester after developing hydrops fetalis. The results highlighted the possible pitfalls of the current program for early screening of alpha-thalassemia despite the program has been nationwide available for many years. Alphathalassemia is a relatively endemic hereditary hemoglobinopathy in Taiwan and about $5 \%$ of population worldwide carries an alphathalassemia variant. Among Taiwanese, the most frequent subtype is the mutant allele called SEA type, which denotes a specific large segment deletion involving consists of both $\alpha$-globin genes (cis, - _SEA ) [2]. Fetuses with hydrops fetalis due to Hb Bart's disease (- - /- -), also called alpha-thalassemia major, usually sustain severe hypoxia since the early stage of organogenesis and cause variety of congenital malformations, maternal complications (such as mirror syndrome and therefore high-risk pregnancies) and neonatal death. As a result, accurate prenatal screening for alpha-thalassemia major is required to prevent those complications. A number of prenatal screening strategies were proposed. Owing to a misunderstanding of the disease or pitfalls of the each test, not all couples who are at risk of conceiving fetuses with $\mathrm{Hb}$ Bart's disease can be identified early by a simple test of MCV during the first antenatal examination, which resulted in a fact that some affected fetuses remained un-diagnosed until late in gestations. In the two cases presented here, the clinicians made several mistakes: 1) MCV of the husband needs to be checked when microcytic anemia was noted in the pregnant woman; and 2) when both the husband and the pregnant woman herself had microcytic anemia, genetic testing of $\alpha$ - and $\beta$-globin genes should be performed in addition to electrophoresis as well as the survey for iron deficiency anemia. Below, we reviewed the current objective methods used to screening for $\mathrm{Hb}$ Bart's hydrops fetalis, including $\mathrm{CBC}, \mathrm{Hb}$ analysis and electrophoresis, genetic testing and ultrasound. Besides, we discussed the limitations of each methodology (Table 1).

\section{Complete cell count}

The commonest screening test for thalassemia is maternal CBC with red blood cell indices as it is easy to perform and readily available. When $\mathrm{MCV}<80 \mathrm{fL}$ or mean corpuscular hemoglobin $(\mathrm{MCH})<25$ $\mathrm{pg} / \mathrm{cells}$ in the absence of iron deficiency, it suggests the case would be carriers of alpha- or beta-thalassemia. Subsequent screening for husband's red blood cell indices and further maternal $\mathrm{Hb}$ analysis are indicated to establish a diagnosis [4]. However, there is a small overlap with normal values in $\alpha^{+}$-thalassemia heterozygotes $(\alpha \alpha / \alpha-)$. In addition, both iron deficiency anemia and beta-thalassemia are also associated with mild to moderate microcytic anemia and frequently result in incorrect diagnosis. Up to today, there is no optimal single marker to discriminate iron deficiency anemia and thalassemia [5]. Thus, serial combined tests to exclude the possibility of iron deficiency anemia and beta-thalassemia are required. Notably that the husband whose MCV was checked should be the biological father of the fetus, otherwise the risk of a false-negative result would exist.

\section{Hemoglobin electrophoresis}

Although $\mathrm{Hb}$ electrophoresis is a simple, rapid, and inexpensive method, it is conducted a screening program for beta-thalassemia when cases have microcytic anemia and elevation of $\mathrm{Hb} \mathrm{A}$, shown in $\mathrm{Hb}$ pattern. It has a few limitations that a significant number of $\mathrm{Hb}$ variants co-migrate with $\mathrm{HbS}$ and $\mathrm{HbC}$ and thus decreases the utility of the method in the definitive identification of variant HbS. Meanwhile, the test cannot define the diagnosis of alpha-thalassemia, especially when coexistence of alpha- and beta-thalassemia. Individuals with iron deficiency anemia or co-inheritance of beta- and alpha-thalassemia mutation in a double heterozygous state would demonstrate decreased or normal the level of HbA2 [6]. Thus, when couples have microcytic anemia and have a normal level of $\mathrm{HbA} 2$, particularly in individuals of SEA or Mediterranean ancestry, alpha-thalassemia carrier can be rationally suspected. Genetic testing for $\alpha$-globin gene is essential to establish the diagnosis.

\section{Genetic testing}

The only certain way to determine the alpha-thalassemia genotype of a suspicious individual is genetic testing of the globin genes. Despite the test is laborious and costly, it can identify the number of loci
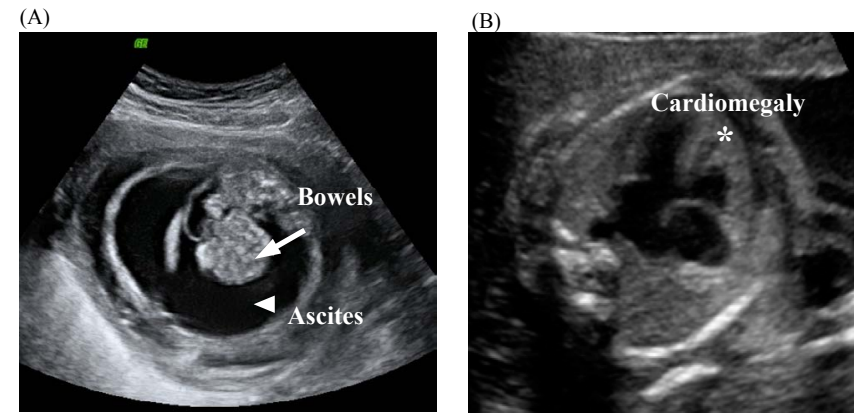

Figure 1: Sonography of a fetus (Case 1 at $\mathrm{GA}=20+6$ weeks) with hemoglobin $(\mathrm{Hb})$ Bart's hydrops fetalis shows $(\mathrm{A})$ massive fetal ascites (triangle) around the echogenic bowels (arrow), and (B) cardiomegaly (star). 
Citation: Wu WJ, Ma GC, Wu PC, Huang KS, Liu YL, et al. (2015) Lessons Learned from Two Missed Prenatal Cases of Hemoglobin Bart's Hydrops Fetalis Until Second Trimester Despite a Nationwide Screening Program. Genetics S7: 003. doi:10.4172/2161-1041.S7-003

Page 3 of 3

\begin{tabular}{|c|c|c|}
\hline Tool & Abnormal Finding & Pitfall \\
\hline \multirow{2}{*}{ Cell count } & $M C V<80 \mathrm{fL}$ or & 1. MCV may be normal for silent carrier ( $\alpha \alpha / \alpha-)$. \\
\hline & $\mathrm{MCH}<25 \mathrm{pg} /$ cells & 2. Falsely normal when combination with iron deficiency anemia or beta-thalassemia. \\
\hline Maternal $\mathrm{Hb}$ analysis & Abnormal level of $\mathrm{Hb}$ pattern & $\begin{array}{l}\text { The level of } \mathrm{HbA}_{2} \text { is decreased or normal when iron deficiency anemia or co-inheritance of alpha- and } \\
\text { beta-thalassemia occurred. } \mathrm{Hb} \text { analysis may result in a false-negative diagnosis of beta-thalassemia. }\end{array}$ \\
\hline Genetic testing & $\begin{array}{l}\text { Deletion or mutation on the loci of } \\
16 \mathrm{p} 13.3\end{array}$ & $\begin{array}{l}\text { Rare non-deletion mutant alleles such as } \mathrm{HbCS}, \mathrm{HbQS}, \mathrm{HbG}-\text { Taichung may escape being detected by } \\
\text { PCR }\end{array}$ \\
\hline \multirow{4}{*}{ Ultrasound } & $\begin{array}{l}\text { 1. Hydrops fetalis (skin edema, pleural } \\
\text { effusion and ascites) }\end{array}$ & 1. The sonographic features usually not appear until the late second trimester. \\
\hline & 2. CTR $\geqq 0.5$ before GA 17 weeks & 2. The sensitivity of CTR is $66.7-100 \%$. Most of the studies are conducted before GA 18 weeks. \\
\hline & 3. CTR $\geqq 0.52$ after GA 18 weeks & \multirow{2}{*}{$\begin{array}{l}\text { 3. MCA-PSV is more effective in prediction of moderate to severe anemic cases in Rhesus } \\
\text { isoimmunization than alpha-thalassemia major. Consequently, diagnosis of Hb Bart's hydrops fetalis } \\
\text { by ultrasound alone may be delayed. }\end{array}$} \\
\hline & $\begin{array}{l}\text { 4. MCA-PSV }>1.5 \mathrm{MoM} \\
\text { Vmax }>95 \text { centile }\end{array}$ & \\
\hline
\end{tabular}

MCV: Mean Corpuscular Volume; MCH: Mean Corpuscular Hemoglobin; HbCS: Hb Constant Spring; HbQS: Hb Quong Sze; CTR: Cardiothoracic Ratio; GA: Gestational Age; MCA-PSV: Peak Systolic Velocity of Middle Cerebral Artery; MoM: Multiples of Median; IHUV Vmax: Intrahepatic Umbilical Venous Maximum Flow Velocity. Table 1: Comparison and limitations of current methodologies used to screen alpha-thalassemia, including hemoglobin (Hb) Bart's hydrops fetalis.

deleted, whether the deletions are in the cis (both copies deleted from one chromosome) or trans (one copy deleted from each chromosome) configuration in individuals with two loci deleted, and any mutations causing non-deletion forms of alpha-thalassemia. In general, $\mathrm{Hb}$ Bart's hydrops fetalis is caused by deletion of all four a-globin genes but in rare cases, the disease can involve other forms of $\mathrm{Hb}$ variants. For example, $\mathrm{HbH}$ disease (- $\alpha /--)$ is usually not lethal. When $\alpha$-globin involves severe non-deletional variants $\left(\alpha \alpha^{\mathrm{ND}} /--\right)$, particularly in the mutations called Hb Constant Spring (HbCS) or Quong Sze (HbQS), it can occasionally cause the $\mathrm{Hb}$ Bart's hydrops fetalis syndrome. Hence, genetic testing is the gold standard [1]. There are now many different polymerase chain reaction (PCR)-based techniques available for the most common alpha-thalassemia mutations [7]. For example, GAP-PCR using specific primers flanking the deletion break-points can detect deletions associated with alpha-thalassemia. Amplification refractory mutation system (ARMS) PCR and Sanger sequencing can identify nondeletional point mutations. In our laboratory, a multiplex PCR panel targeted to 10 common $\alpha$-globin mutations is routinely used for rapid alpha-thalassemia genotyping of Taiwanese patients (Table 2).

\section{Ultrasound features}

Once the pregnancy affected with $\mathrm{Hb}$ Bart's hydrops fetalis or $\mathrm{HbH}$ disease progresses, several sonographic features would become apparent, including cardiomegaly, placentomegaly, hepatosplenomegaly and hydrops fetalis (skin edema, pleural effusion or ascites). However, in most of the time, not all these classical sonographic features would appear until the late second trimester [8]. In the first trimester, cardiovascular compensation secondary to alpha-thalassemia major may take place initially. Lam and colleagues [9] described the predictive value of the cardiothoracic ratio for alphathalassemia major in 1997. It should be measured under the section of 4-chamber view; a standard view should show two groups of atrioventricular valves, one complete rib, and three strong echogenic foci of the spine. The maximum cardiac is measured at end diastole phase at the level of annulus. The cardiothoracic ratio is calculated as the ratio of the cardiac diameter to the thoracic diameter (Figure 2) [10]. A cardiothoracic ratio greater than 0.5 before GA 17 weeks or 0.52 after GA 18 weeks is considered abnormal. Although some discrepancy existed among studies reported, all of them showed that using the cardiothoracic ratio as a predictor of alpha-thalassemia major had high sensitivity $(66.7-100 \%)$ and specificity $(92-100 \%)$, whereas the falsepositive rate $(0-9.1 \%)$ was low.

For anemic fetuses resulting from Rhesus isoimmunization, anemia can be detected by Doppler ultrasonography on the basis of an increase in MCA-PSV. Mari et al. [11] reported that a MCA-PSV of greater than $1.5 \mathrm{MoM}$ could be an indicator for screening moderate to severe fetal anemia with sensitivity nearly $100 \%$ (95\% confidence interval, 0.86-1.0) and the false-positive rate of 12\%. In 2009, Pretlove et al. [12] performed meta-analysis on the diagnostic value of MCA Doppler flow studies for fetal anemia. The sensitivity dropped to $75.5 \%$ and the specificity was $90.8 \%$ for detecting severe anemia. Although fetal MCA-PSV is considered the best noninvasive marker in Rhesus isoimmunization, there is no enough data demonstrating that MCAPSV is as effective in predicting alpha-thalassemia major. Leung et al. [13] reported that using MCA-PSV as a parameter in prediction of alpha-thalassemia major during GA 16-20 weeks, the diagnosis may be delayed in $25 \%$ of affected pregnancies. Furthermore, by the combination of cardiothoracic ratio (CTR) and MCA-PSV, it tended to increase the sensitivity at GA $12-15$ weeks $(100 \%)$ and decreased the false positive rate at GA 16-20 weeks (0\%). Hence, screening for $\mathrm{Hb}$ Bart's hydrops fetalis by ultrasound parameters alone may be a choice, despite not satisfactory, to at-risk patients who decline invasive procedures.

In addition to MCA-PSV, Leung et al. [14] presented that intrahepatic umbilical venous maximum flow velocity (IHUV Vmax) was also a useful parameter in diagnosis and management of alphathalassemia major fetuses, when their cases demonstrated the consistent declines in value after receiving intrauterine transfusion.

\section{Fetal therapy for hydrops fetalis}

Intrauterine transfusion seems to prolong neonatal lifespan and minimize the complications. However, the procedure usually started after completion of organogenesis. The fetuses are undoubtedly at high risk for major neurodevelopment problems and congenital anomalies. Furthermore, lifetime transfusion therapy or stem cell transplantation is required after their birth. Hence, a careful and detailed non-directive genetic counseling should be offered at prenatal stage whenever termination of the pregnancy is still possible [15].

\section{Conclusion}

Alpha-thalassemia is an old disease with available nationwide screening programs. However, it remains a challenging task to screen all the at-risk couples and make a definitive diagnosis in early pregnancy, even in a country like Taiwan having an excellent medical standard and a comprehensive nationwide screening program for more than 20 years. The presence of microcytic anemia is a major clue and thus microcytic anemia is part of the initial evaluation in routine prenatal and preconceptional screening. Nevertheless, there are still a few cases 
Citation: Wu WJ, Ma GC, Wu PC, Huang KS, Liu YL, et al. (2015) Lessons Learned from Two Missed Prenatal Cases of Hemoglobin Bart's Hydrops Fetalis Until Second Trimester Despite a Nationwide Screening Program. Genetics S7: 003. doi:10.4172/2161-1041.S7-003

Page 4 of 3

\begin{tabular}{|c|c|c|c|}
\hline Mutation type & Mutant allele & HGVS nomenclature & Diagnostic method \\
\hline \multicolumn{4}{|c|}{ 1. Deletions } \\
\hline \multirow[t]{5}{*}{ Alpha-thalassemia 1} & - - SEA & NG_000006.1:g.26264_45564del19301 & GAP-PCR \\
\hline & - _FIL & NG_000006.1g.11684_43534del31851 & GAP-PCR \\
\hline & - - THAI & NG_000006.1:g.10664_44164del33501 & GAP-PCR \\
\hline & - -MED & NG_000006.1:g.24664_41064del16401 & GAP-PCR \\
\hline & $-(\alpha)^{20.5}$ & NG_000006.1:g.15164_37864del22701 & GAP-PCR \\
\hline \multirow[t]{2}{*}{ Alpha-thalassemia 2} & $-a^{3.7}$ & NG_000006.1:g.34164_37967del3804 & GAP-PCR \\
\hline & $-\alpha^{4.2}$ & HGVS not attributable & GAP-PCR \\
\hline \multirow[t]{3}{*}{ 2. Non-deletion point mutations } & $\mathrm{HbCS}$ & HBA2:c.427T>C & ARMS-PCR \\
\hline & $\mathrm{HbQS}$ & HBA2:c.377T>C (or HBA1) & ARMS-PCR \\
\hline & -aG-Taichung & HBA1:c.223G>C (p.D75H) & ARMS-PCR \\
\hline
\end{tabular}

HGVS: Human Genome Variation Society; ARMS: Amplification Refractory Mutation System.

Table 2: A multiplex PCR panel targeted to 10 common a-globin mutations is available for rapid alpha-thalassemia genotyping of Taiwanese patients.

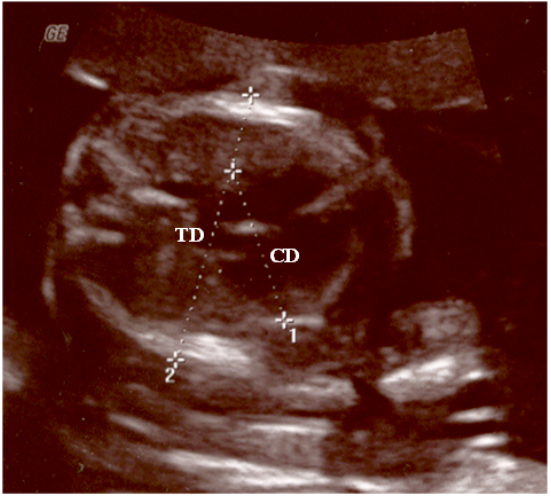

Figure 2: The cardiothoracic ratio (CTR) is measured as the ratio of cardiac dimension (CD) to thoracic dimension (TD) at the level of 4 chamber view.

of fetus with $\mathrm{Hb}$ Bart's hydrops fetalis remained un-diagnosed until late pregnancy. Nowadays, with the development of target sequencing of maternal plasma DNA, it is feasible to detect fetal monogenic disease by noninvasive prenatal diagnosis. Lam et al. [16] successfully applied the method to determine homozygous beta-thalassemia prenatally. In addition, preimplantation genetic diagnosis is also a feasible option for at-risk couples that do not accept a 1 in 4 risk of having an affected fetus and the subsequent suffering related to abortions and termination of pregnancy [17]. Physicians should understand the benefits and disadvantages and limitations of each screening test in order to offer appropriate preconceptional counseling and prenatal management.

\section{References}

1. Piel FB, Weatherall DJ (2014) The a-thalassemias. N Eng J Med 371: 19081916.

2. Chang JG, Liu HJ (1995) Molecular diagnosis of thalassemia in Taiwan. Kaohsiung Journal of Medical Sciences 11: 371-373.

3. Weatherall DJ (1995) The hemoglobinopathies. In: The Metabolic and Molecular Bases of Inherited Disease. (7thedn), McGraw-Hill, New York.

4. Lafferty JD, Barth DS, Sheridan BL, McFarlane AG, Halchuk LM, et al. (2007) Prevalence of thalassemia in patients with microcytosis referred for hemoglobinopathy investigation in Ontario: a prospective cohort study. Am J Clin Pathol 127: 192

5. Schoorl M, Schoorl M, van Pelt J, Bartels PC (2015) Application of innovative hemocytometric parameters and algorithms for improvement of micro cystic anemia discrimination. Humatol Rep 7: 5843

6. Denic S, Agarwal MM, Al Dabbagh B, El Essa A, Takala M, et al. (2013) Hemoglobin A2 Lowered by Iron Deficiency and $\alpha$-Thalassemia: Should Screening Recommendation for $\beta$-Thalassemia Change? ISRN Hematol 2013: 858294.
7. Old J, Traeger-Synodinos J, Galanello R, Petrou M, Angastiniotis M (2012) Prevention of thalassemias and other hemoglobin disorders. (2 edn), Nicosia Cyprus: Thalassemia International Federation.

8. Tongsong T, Wanapirak C, Srisomboon J, Piyamongkol W, Sirichotiyakul S (1996) Antenatal sonographic features of 100 alpha-thalassemia hydrops fetalis fetuses. J Clin Ultrasound 24: 73-77.

9. Lam YH, Ghosh A, Tang MH, Lee CP, Sin SY (1997) Early ultrasound prediction of pregnancies affected by homozygous alpha thalassemia-1. Prenat Diagn 17: 327-332.

10. Li X, Zhou Q, Zhang M, Tian X, Zhao Y (2015) Sonographic Markers of Fetal a-Thalassemia Major. J Ultrasound Med 34: 197-206.

11. Mari G, Deter RL, Carpenter RL, Ragnab F, Zimmerman R, et al. (2000) Noninvasive diagnosis by Doppler ultrasonography of fetal anemia due to maternal red-cell alloimmunization. Collaborative Group for Doppler Assessment of the Blood Velocity in Anemic Fetuses. N Engl J Med 342: 9-14.

12. Pretlove SJ, Fox CE, Khan KS, Kilby MD (2009) Noninvasive methods of detecting fetal anaemia: a systematic review and meta-analysis. BJOG 116 : 1558-1567.

13. Leung KY, Cheong KB, Lee CP, Chan V, Lam YH, et al. (2010) Ultrasonographic prediction of homozygous alpha0-thalassemia using placental thickness, fetal cardiothoracic ratio and middle cerebral artery Doppler: alone or in combination? Ultrasound Obstet Gynecol 35: 149-154.

14. Leung WC, Oepkes D, Seaward G, Ryan G (2002) Serial sonographic findings of four fetuses with homozygous alpha-thalassemia-1 from 21 weeks onwards. Ultrasound Obstet Gynecol 19: 56-59.

15. Chmait RH, Baskin JL, Carson S, Randolph LM, Hamilton A (2015) Treatmen of alpha(0)-thalassemia (--(SEA)/--(SEA)) via serial fetal and post-natal transfusions: Can early fetal intervention improve outcomes? Hematology 20 : 217-222.

16. Lam KW, Jiang P, Liao GJ, Chan KC, Leung TY, et al. (2012) Noninvasive Prenatal Diagnosis of Monogenic Diseases by Targeted Massively Paralle Sequencing of Maternal Plasma: Application to $\beta$-thalassemia. Clin Chem 58 : 1467-1475.

17. Chen M, Chan JK, Nadarajah S (2015) Single-tube nonaplex microsatellite PCR panel for preimplantation genetic diagnosis of $\mathrm{Hb}$ Bart's hydrops fetalis syndrome. Prenat Diagn 35: 534-543.

This article was originally published in a special issue, Genetic Diseases handled by Editor(s). Dr. Steven J. Fliesler, Buffalo VA Medical Center, USA; Dr. Jijing Pang, University of Florida, USA 